\title{
The Impact of Multi-sensory Stimuli on Confidence Levels for Perceptual-cognitive Tasks in VR
}

\author{
Sungchul Jung* \\ Human Interface Technology Lab NZ
}

University of Canterbury
Andrew L. Wood ${ }^{\dagger}$

Human Interface Technology Lab NZ

University of Canterbury
School of Product Design

University of Canterbury
Robert W. Lindeman ${ }^{\text {II }}$

\author{
Simon Hoermann \\ School of Product Design \\ University of Canterbury
}

Human Interface Technology Lab NZ

University of Canterbury

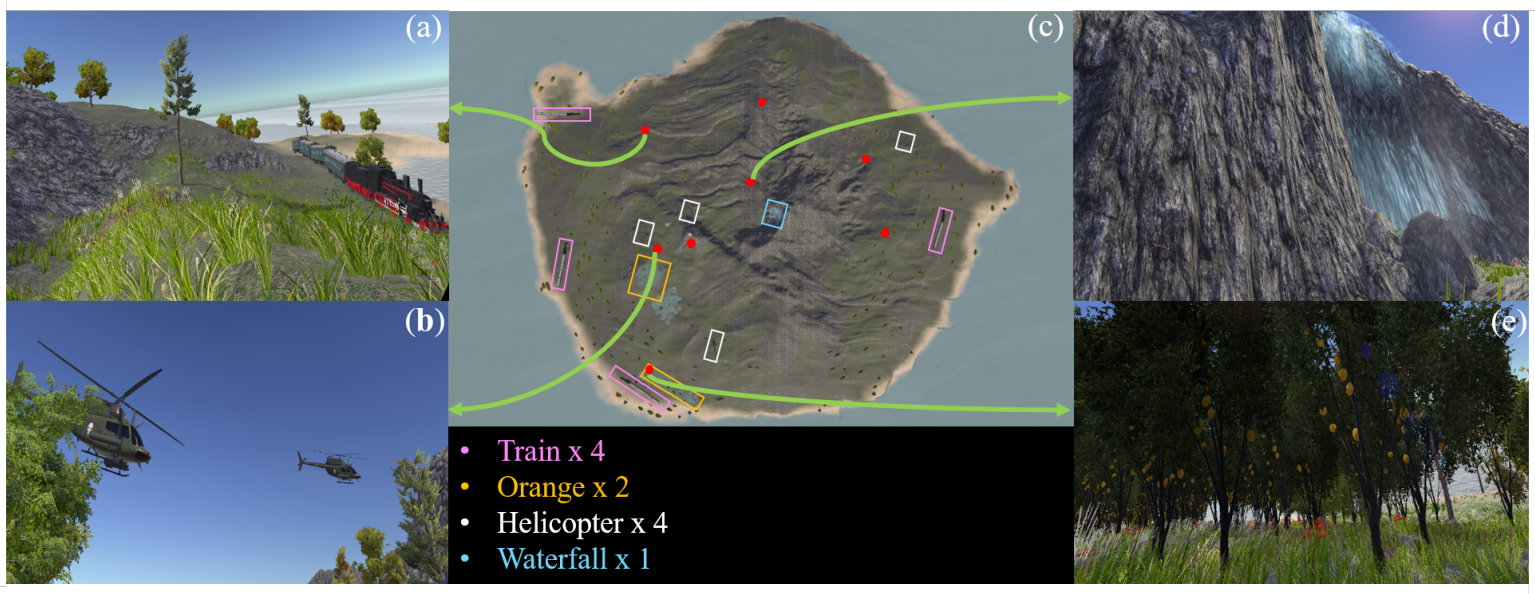

Figure 1: Top view of the virtual environment, with call-outs for four out of the eight locations on the map. For each trial, the user first received a textual description of a location. Then, on the island (c), the user visited one of eight different places with different types of sensory feedback. We designed the island with particular scene features, including trains (boxed in pink on the map), orange trees (yellow), helicopters (white), and a waterfall (blue). We mapped sensory feedback (vision, audio, wind, floor vibration, and smell) to the scene features and varied them, depending on the study condition.

\section{Abstract}

Supporting perceptual-cognitive tasks is an important part of our daily lives. We use rich, multi-sensory feedback through sight, sound, touch, smell, and taste to support better perceptual-cognitive things we do, such as sports, cooking, and searching for a location, and to increase our confidence in performing those tasks in daily life. Same with real life, the demand for perceptual-cognitive tasks exists in serious VR simulations such as surgical or safety training systems. However, in contrast to real life, VR simulations are typically limited to visual and auditory cues, while sometimes adding simple tactile feedback. This could make it difficult to make confident decisions in VR.

In this paper, we investigate the effects of multi-sensory stimuli, namely visuals, audio, two types of tactile (floor vibration and wind), and smell in terms of the confidence levels on a location-matching task which requires a combination of perceptual and cognitive work inside a virtual environment. We also measured the level of presence when participants visited virtual places with different combinations of sensory feedback. Our results show that our multi-sensory VR

\footnotetext{
*e-mail: sungchul.jung@ canterbury.ac.nz

†e-mail: ali66@uclive.ac.nz

†e-mail: simon.hoermann@canterbury.ac.nz

§e-mail: pram.abhayawardhana@canterbury.ac.nz

Ile-mail: gogo@hitlabnz.org
}

system was superior to a typical VR system (vision and audio) in terms of the sense of presence and user preference. However, the subjective confidence levels were higher in the typical VR system.

Keywords: Multisensory VR, Perception, Confidence, Cognition, Floor vibration, Wind, Smell

Index Terms: Human-centered computing-Virtual reality; Computing methodologies-Perception; Hardware-Sensor devices and platforms; Human-centered computing-User studies; Humancentered computing-User interface design;

\section{INTRODUCTION}

In our daily lives, we frequently confront situations where the perceptual-cognitive load is increased, be it a routine or important one. How people make decisions, and what factors influence the decision process, is a critical issue [10]. For better performance on perceptual-cognitive tasks, Christopher et al. [56] state that decisions should be made after accounting for all of the available information, and this is the decision procedure that should also followed for virtual reality (VR) situations, since the sophistication of current systems has reached such a high level. For VR simulation of serious, real-world problem scenarios, such as surgery or healthand-safety training, users often wear high-resolution head-mounted displays (HMDs) and interact with the virtual world using supportive devices [26]. It is now possible to experience VR using highly immersion technologies. Others have also conducted studies on how users handle perceptual-cognitive tasks using highly immersive VR environments in various scenarios, including medical training, extreme ethical situations, and firefighter training [7, 46, 53]. However, 
most studies have focused on the decision itself, without considering the user's subjective confidence in his or her decisions. How accurate the user's certainty is regarding their decision can be affected by the amount and type of information they use [28]. From this perspective, previous studies created VR experiences using visual and audio feedback only [46,53], or perhaps added tactile feedback (vibration) [7], and depended on this partial-sensory information. It is still unclear, however, what the impact of extended sensory dimensions are on user confidence in terms of decision making.

To address this question, we implemented a multi-sensory VR (MVR) system that extended the sensory dimensions beyond visual and audio feedback. MVR enables us to deliver two types of tactile cues (floor vibration and wind), as well as smell feedback. With the system, we conducted a study into the effects of MVR in terms of confidence level and correct answer ratio as objective measurements, and sense of presence, user preference, and cybersickness as subjective measurements in VR by comparing our MVR system to a typical-sensory VR system (TVR) supporting only visual and audio feedback. In this experiment, the participants received a binary question (Yes/No) and reported their answer, as well as a confidence level using the VR controller. The task was to determine if a visited virtual location matched a previous description of a place. The participant visited eight virtual places on a virtual island (Figure 1c), marked with red dots, multiple times. Each virtual location had uniquely designed features (Figure 1a,b,d,e) which explicitly (visual, audio) or implicitly (floor vibration, wind blowing, scent) represented more sensory feedback. Depending on the condition, the participant experienced virtual locations with MVR or TVR. For this study, our focus was more about the perceived confidence level for the answer in perceptual-cognitive task scenarios, and less about the answer itself. Thus, we created a simple task with low complexity and expected performance regarding the answer would be similar in both conditions, but that the confidence level would be different. We describe the details in Section 3.

Our results show that MVR is superior to TVR in terms of sense of presence and user preference, but that confidence levels were higher in TVR for our specific task. More details will be given in Section 4.

The paper is structured as follows. Section 2 gives an overview of related work. Section 3 describes the experiment that we conducted to investigate the impact of MVR. Section 4 presents the results, with associated discussions in Section 5. Section 7 concludes the paper and suggests future research directions.

\section{Related Work}

\subsection{Multi-sensory VR}

The augmentation of virtual experiences with cues beyond the traditional visual and auditory stimuli has been the center of research for some time. In therapeutic applications, the efforts were often to substitute one sensory system with another to overcome limitations of some users related to one sensory modality e.g., to provide tactile feedback or scent feedback to compensate for the lack of eyesight [1] or to enhance non-digital film experiences [23]. Recent research, such as a study by Kruijff et al., suggests that it is possible to enhance user experiences in virtual environments through the use of multi-sensory cues $[38,39]$. Feng et al. explored the use of multi-sensory feedback that combines floor vibration feedback with wind feedback provided through ventilator fans [14-16]. Multisensory VR was also suggested as a tool to collaboratively author experiences in real-time [8]. The concept of multi-sensory VR was also picked up for tourism and marketing, where, for example, it was suggested as a use for creating memorable experiences that help the marketing of a local wine and hence increase its international competitiveness [43]. Manghisi et al. pointed out that VR could have strong impact for showcasing a region's attractions [42]. They designed MATE, Multi-sensory Apulia Touristic Experience, a multi-sensory screen-based system that can be packed in a container and transported around Europe to virtually showcase and promote Apulia, a region of Italy.

\subsubsection{Tactile Feedback}

Vibration feedback was suggested to be useful to enhance the accessibility of games for people with impaired vision $[57,58]$. Others have tried to use vision to fool people into experiencing tactile sensations in real life, as well as in mediated reality $[3,25,48]$. However, in this paper we focus on the use synchronized multi-sensory cues to augment basic visual and auditory feedback with the aim of enhancing the overall user experience.

Several ways of integrating haptic feedback into a virtual reality system have been explored in the past. In many cases, feedback systems were part of the user interface controls, such as integrated force feedback in a joystick or a mechanical tracking system, or vibration feedback in gamepads. Also, wearable systems such as vibration vests or vibration-enhanced headphones $[6,40,41]$ were developed. In addition, force feedback exoskeletons were suggested in the literature to provide additional sensory information to the user, for example to enhance the sensation of grasping an object in VR [18, 20]. In recent years, research into external vibration feedback started to become more popular. Kruijff et al. for example experimented with bass-shakers and found that the point of entry i.e., the body-part closest to the vibration device, gets the most stimulation, however it is also possible to tailor the experience to other parts of the body by delivering different vibration frequencies, due to differences in resonance frequencies of different body structures [39].

\subsubsection{Scent Feedback}

Olfaction is used by humans and animals as one of the most important sensory channels for gaining a full understanding of their environments. Different odorants are related to physiological, behavioral, and psychological changes in humans, which are associated with olfactory memory $[17,24]$. Therefore, many recent studies have used olfaction as a method of modifying certain experiences for humans in a variety of different applications $[2,12,21,59,61]$. The use of olfactory displays in the development of notification systems has been studied by many researchers. Dobbelstein et al. developed a wearable olfactory display that could be worn as a pendant around the neck, allowing the user to receive personal scented notifications as a channel by amplifying received cell phone notifications with artificially emitted scents [12]. Another group compared the effect of the use of visual, audio or olfactory displays that delivering notifications had on a user's engagement in a cognitive task [2]. Other work related to odor delivery with respect to multimedia systems was done by Kaye in his personal-notification alarm applications [35], and Brewster, McGookin and Miller with olfactory displays used for multimedia content searching, browsing and retrieval, to aid in the search of digital photo collections [5]. Although numerous prior studies have been conducted using olfactory displays integrated with other media objects in VR $[9,11,13,21,27,30,35,45,49,61]$ only a few show strong evidence of how olfactory cues impacted considerably on the sense of reality of the user in the application [21, 27, 61].

The unique properties of olfaction make smell-based interaction a promising tool to enhance experiences in VR. Heilig used odor emitters among other modalities in his Sensorama, which is considered to be one of the earliest VR systems to enhance the user experience of a motion picture [23]. Olfaction-enhanced multimedia is a challenging research area, due to the complexity of the sense of olfaction, as well as the struggle of implementation and control of the variables $[21,27]$. Examples of some challenges include the difficulty in controlling odorant delivery and the challenge of delivering multiple scents to the user at a precise time and space without interrupting the user or contaminating the environment $[44,50]$. Another concern is perfect synchronisation of the delivery of the odorant with 
other stimuli. Creating an effective odorant delivery system, which is easy to use and control, is another area that needs improvement. Moreover, high intensities of certain odorants used in experiments also tend to lead to a variety of undesirable reactions (allergies, sensitivities) in the subjects. Therefore, having an appropriate intensity of a carefully formulated odorant delivery solution affects the overall quality of the results.

Odorant delivery systems developed for some studies as reported in the literature suffer from limitations such as having to wear special accessories $[29,44,52,59]$ designed to deliver the odorant, introducing additional work, noise or tactile stimulation to the skin of the user, disturbing the overall immersive experience. These accessories, such as scented collars, helmets, masks and other attachments, can also complicate the study when body movement is restricted. Although Yanagida et al. proposed an odor delivery device that involved an "air cannon" which projects scented air puffs near the user's nose without requiring the user to attach anything on the head, this method increased the odor contamination in the air [62]. In the present study, we include olfaction in the VR environment using three different odorants that are familiar to most people, clearly distinguishable from each other, and easily detectable. The odor materials were formulated cautiously to deliver a controlled amount of scent to each participant considering the relative odor impact of each odorant, as well as the safety of the participants. Furthermore, a deodoriser was used in the study between the deliveries of different odorants to avoid the likelihood of contaminating the ambient air.

\subsection{Immersive VR}

The level of immersion and presence are critical measurement in VR. Immersion refers to the objective level of sensory fidelity a VR system provides. Technical support, such as a high-resolution HMD with a wide field of view and 3D tracking system, form a high level of immersion, and can produce a sense of presence, which indicates a psychological response toward to a VR system $[4,55]$ While the level of immersion depends on the system capability objectively, Slater et al and Usoh et al. [54,60] developed a subjective questionnaire to measure the sense of the presence and researchers actively use it $[31-33,51]$. As another indicator for the subjective feeling towards the immersion, Ye and Steed [63] considered a sense of virtual body ownership illusion, a self-consciousness of one's own body, and Kilteni et al. [37] extended the idea with multi-sensory feedback. In this paper, we hypothesize the illusion could occur because of synchrony, and thus, having more cues depending on body motion can reasonably be expected to increase presence. With a similar mechanism, having more syncronized cues on the multiple sensory feedback channels can be expected to increase presence. Thereby, we measure presence to investigate the impact of multisensory VR in this paper.

\section{Methods}

In this section, we describe the experiment that we conducted to investigate the impact of multi-sensory virtual reality system on the confidence level, sense of presence, user preference, and cybersickness in virtual reality. Our goal is to explore the impact of the simultaneously delivered multiple sensory feedback, we did not focus on the level of intensity of each sensory stimulus thereby we decided to use empirically adopted intensity level for each sensory stimulus.

\subsection{Pilot Study}

Empirically deciding on an effective strength of a formulated odor is not intuitive compared to other sensory modalities due to the complex human nasal system, air-conditioning, and individual health conditions. To minimize odor detecting error, we conducted a pilot study with 10 male and 6 female participants (age $M=19.9, S D=5.69$ ) to decide the odorant intensity level. For this pilot study, we used the full experimental setup and procedure. Since we will be using other sensory feedback including fans, we had to consider the physical environment. After the pilot study, we reformulated and confirmed the scent intensity, and conducted the actual experiment with predefined vision, audio, tactile (floor vibration and wind) and smell feedback in the identical experimental setup and procedure.

\subsection{Participants}

Before recruiting participants, we conducted an a priori power analysis to compute the required sample size using $G^{*}$ Power [19]. For a large effect size with a power of 0.8 , we determined the need for a minimum of 15 participants with our study design. Based on the feedback from the pilot study, we revised the scent strength and conducted the actual experiment by recruiting six male and 11 female participants (age $M=23.9, S D=8.31$ ). Our analysis reported here is based on these 17 subjects.

We had to exclude one participant's data from the analysis due to a logging problem. We recruited participants using on-campus fliers at the local university. Participants received a small monetary compensation for their participation

\subsection{Material}

We provided the high-level architecture for the system in Fig 2. We implemented a client and server model as a basic structure. A client computer worked for rendering VR environments on Unity 3D engine, data logging, system control and dispatching command to the server for running each sensory components. We used HTC Vive with an Intel computer with core i7 CPU and NVIDIA GeForce GTX $1080 \mathrm{GPU}$ and 32GB of RAM. To reduce the expected latency and manipulating the sensory component efficiently, we implemented an independent server computer with an Intel computer with core i7 CPU and 32GB of RAM. This server computer handles interfacing with the received command from the client and running or stopping the sensory devices for fans, aroma diffusers and vibrators underneath the floor.

\subsubsection{Multi-sensory System}

The system features floor vibration feedback, wind feedback and scent feedback matching the visual and audio feedback. We used brushless DC fans (Delta Electronics Inc., model: AFB1212SHE), ButtKicker LFE and Soehnle USB Aroma Diffusers to provide wind, vibration and scent feedback, respectively.

We provided wind feedback via an setup of 16 fans arranged in a ring around the cage facing inwards on two levels (Fig 3). Each level had eight fans and was offset from the other ring to provide a staggered arrangement. We controlled the speed of each fan by passing a value between 0 and 255

The odorant delivery feedback was provided using a combination of a formulated odor (essential oils) delivery solution and a USB stick connected to a USB port (See Fig 3). The USB stick was plugged in to a hub that supported toggling the power to each port individually. Once powered, the USB stick would heat up a thick cotton absorbent pad layered over a heating coil inside the USB stick. The absorbent layer was treated with a measured amount of the formulated odorant delivery solution. The USB hub was attached to one of the fans facing the participant, and was controlled to deliver the individual odorants by providing power (for five seconds) to the respective USB port. When a certain port on the hub attached behind the fan was powered, the odorant liquid, which was absorbed into the pad, was consistently vaporized and delivered to the participant through the controlled airflow created by the fan. We chose a fanbased diffusing system since it delivers the scent feedback naturally along with our study scenario context (a task in a wild environment), even though a stimulus-onset delay of about a second was present.

We generated the floor vibration using four low frequency transducers attached to the underside of the floor, corresponding to the 


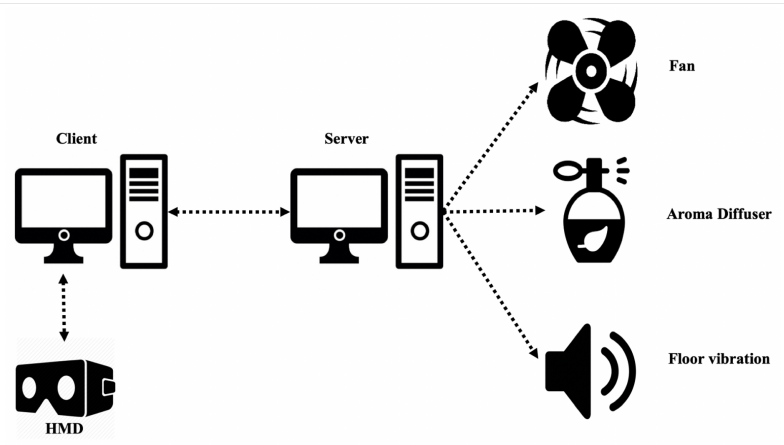

Figure 2: System architecture. The client computer works for rendering VR environments, user interaction, data logging, and communicating with the server computer which handles interfacing with the sensory devices, fans, aroma diffusers and vibrators underneath the floor.

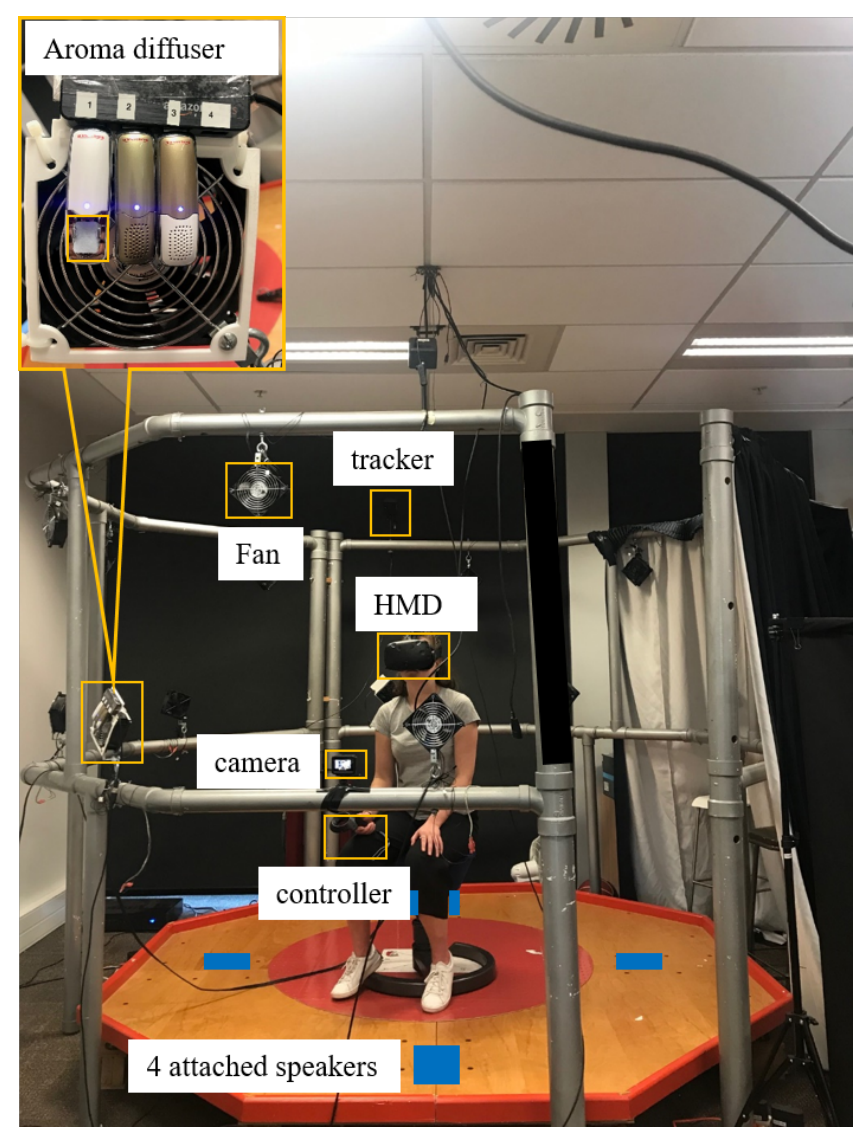

Figure 3: Experimental platform. The participant was sitting on a chair in the middle of the cage with wearing an HMD and holding a controller. Depend on the condition in MVR, the participant received wind blowing from the fans, had smell scent from the aroma diffuser, and felt floor vibration which located in the 4 points (marked with blue color on the floor).

blue squares seen in Fig 3. These transducers receive audio signals from the server via an amplifier and an audio splitter. All four of the transducers thus receive the same audio signal.

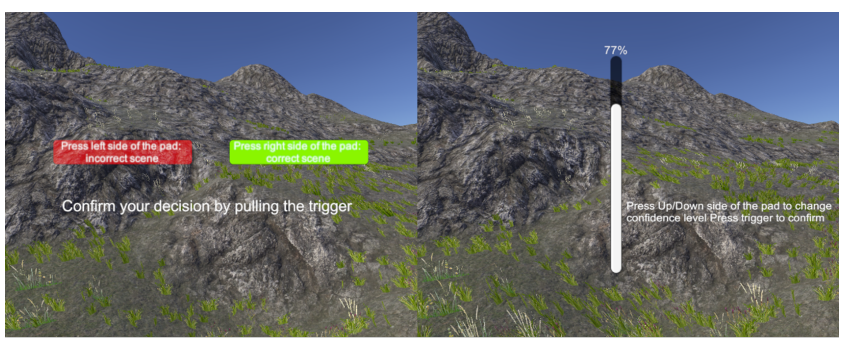

(a)

(b)

Figure 4: Interface for reporting the answer and confidence level. (a) Determine whether or not they were in the scene described. "no" on the left with red color and "yes" on the right with green color. (b) Determine how confident they were in their answer via a vertical bar which could be filled from $0 \%$ to $100 \%$

\subsubsection{Virtual Environment}

Two virtual environments were used in this study: A darkened scene used for instructing and providing descriptions to the subject and a virtual island used as the scene of the experiment. The island scene contained four trains, two orange trees, four helicopters and a waterfall scattered across it (See Fig 1). This allowed the participant to be presented with multiple different scenes using the same environment.

We provided two stages of the input interface to the participants. In the first stage, we presented "Confirm your decision by pulling the trigger" to asked the user to determine whether or not they were in the scene described to them. We located a "no" button on the left with the red color and located a "yes" button on the right with the green color that corresponded to the areas on the controller. The participant had to press the left or right region of the touchpad on the controller to give the answer and confirmed it by pulling the trigger button (See Fig 4 (a)). After giving the answer, in the second stage we showed "press Up/Down side of the pad to change confidence level. Press trigger to confirm." to asked the user to determine how confident they were in their decision via a vertical bar which could be filled from $0 \%$ to $100 \%$ using the up or bottom region of the touchpad on the controller and confirmed it by pulling the trigger button again (See Fig 4 (b)).

\subsection{Study Design}

We used a within-subject design with one independent variable, $V R$ system (Multi-sensory VR, Typical-sensory VR) with six dependent variables that are confidence level, consumed time for the giving answer, and correct answer ratio as objective measures and sense of presence, cybersickness, and user preference as subjective measures. We used a random order for the experiment including the VR system condition, virtual scene, and scene scenario types to avoid any ordering effect. The experiment was approved by our organization's Human Ethics Committee.

\subsubsection{Scenario}

To investigate the impact of the multi-sensory VR system, we asked the participant to experience several virtual environments that were rendered with two types of VR feedback systems for the same environment. In MVR, we provided vision, audio, tactile (wind blowing, floor vibration), and smell feedback, but only vision and audio feedback in TVR.

Since our major research question was related to user confidence based on the perceptual-cognitive task, we adopted a concept of signal detection theory, which is nearly all reasoning and decision making occurs in the presence of some uncertainty [22], to force the participant to make a decision and report a confidence level for it. We designed this experiment with the two-alternative forced 
Table 1: Sensory Component Combinations: Upper-case letters indicate each sensory modality and the lower-case letters indicate a specific description of the sensory stimulus

\begin{tabular}{|c|c|c|c|c|c|}
\hline & & \multicolumn{2}{|c|}{ MVR } & \multicolumn{2}{|c|}{ TVR } \\
\hline \# & Script & Matched & Mismatched & Matched & Mismatched \\
\hline 1 & $\begin{array}{l}\text { You are in a scene where there are helicopters hover- } \\
\text { ing in the sky. }\end{array}$ & $V_{H}+A_{H}+S_{T}+W_{W}+F_{H}$ & $W_{W}$ & $V_{H}+A_{H}$ & $N_{/} A$ \\
\hline 2 & You are in a scene where there is a train moving. & $V_{T}+A_{T}+S_{T}+W_{B}+F_{T}$ & $W_{B}$ & $V_{T}+A_{T}$ & $N_{/} A$ \\
\hline 3 & $\begin{array}{l}\text { You are in a scene where there are orange trees and } \\
\text { a train moving. }\end{array}$ & $V_{T L}+A_{T}+S_{L}+W_{B}+F_{T}$ & $V_{L}+S_{L}+W_{B}$ & $V_{T L}+A_{T}$ & $V_{L}$ \\
\hline 4 & You are in a scene where there is a water fall. & $V_{W}+A_{W}+S_{W}+W_{W}+F_{W}$ & $W_{W}$ & $V_{W}+A_{W}$ & $N_{/} A$ \\
\hline 5 & $\begin{array}{l}\text { You are in a scene where there are helicopters and a } \\
\text { train moving. }\end{array}$ & $V_{H T}+A_{H T}+S_{T}+W_{W}+F_{H T}$ & $V_{T}+A_{T}+S_{T}+W_{W}+F_{T}$ & $V_{H T}+A_{H T}$ & $V_{T}+A_{T}$ \\
\hline 6 & $\begin{array}{l}\text { You are in a scene where there is a waterfall and a } \\
\text { train moving. }\end{array}$ & $V_{T W}+A_{T W}+S_{T W}+W_{W}+F_{T W}$ & $V_{T}+A_{T}+S_{T}+W_{W}+F_{T}$ & $V_{T W}+A_{T W}$ & $V_{T}+A_{T}$ \\
\hline 7 & $\begin{array}{l}\text { You are in a scene where there is a train moving and } \\
\text { orange trees. }\end{array}$ & $V_{T L}+A_{T T}+S_{T L}+W_{W}+F_{T}$ & $V_{L}+S_{L}+W_{W}$ & $V_{T L}+A_{T T}$ & $V_{L}$ \\
\hline 8 & $\begin{array}{l}\text { You are in a scene where there are helicopters and a } \\
\text { waterfall. }\end{array}$ & $V_{W}+A_{H W}+S_{T W}+W_{W}+F_{H W}$ & $V_{W}+A_{W}+S_{W}+W_{W}+F_{W}$ & $V_{W}+A_{H W}$ & $V_{W}+A_{W}$ \\
\hline
\end{tabular}

Table 2: Forced Choice Design with two scenario pairs. Regarding the probe type (matched sensory or mismatched sensory compared to description), the participant made a response (yes or no)

\begin{tabular}{c||cc} 
& Yes & No \\
\hline \hline Matched & Hit & False negative \\
\hline Mismatched & False positive & Correct reject
\end{tabular}

choice as objective queries which we prepared a description-matched sensory stimulus pair and description mismatched sensory stimulus pair. Thereby the participant made four possible outcomes which are Hit (correct acceptance of a stimulus), Miss or False negative (incorrect rejection of true stimulus), False alarm or False positive (incorrect acceptance of a false stimulus) and Correct reject (correct rejection of false stimulus) (See Table 2). In this paper, we use favor False positive and False negative over the other terms.

For example, after putting on the HMD, each participant was given a description for a particular location in a VE in a darkened virtual space. After the participant confirmed reading the description, they were teleported to a certain location in the VE and were asked to decide if the place matched the description. After giving the answer, the participant returned to the darkened virtual space, confirmed the next description, and was teleported to the next location in the VE. They repeated this process 32 times to complete a study.

\subsubsection{Sensory Component Combinations}

To reduce any unexpected bias from the virtual environment design with a specific sensory stimulus, we generated multiple scenes by combining diverse visual, audio, tactile, and smell feedback. Since it is not feasible to test all the possible cases which are 216 cases in this study, we selected the best combination of the sensory feedback to deliver perceptually matched sensory stimulus among them as much as possible. For designing the VE, we considered two primary sensory feedback (vision and audio) first which are four visual features along with matched three audio resources in TVR. Based on the TVR, we expanded two sensory dimensions with secondary sensory feedback which including two types of tactile and three types of scent in MVR. We described the selected sensory component in this study as below:

- Visual $(V)$ : Helicopter (h), Train (t), orange tree (1), Waterfall with Grasses (w)
- Audio (A): Helicopter (h), Train (t), Waterfall (w)

- Smell $(S)$ : Tar (t), orange (1), Wet grass (w)

- Wind $(W)$ : Breeze (b), Windy (w)

- Floor $(F)$ : Helicopter (h), Train (t), Waterfall (w)

With the selected sensory components, we designed 8 virtual environments using eight scene descriptions for matched scenario and mismatched scenario in MVR and TVR, respectively so that a participant visited a virtual environment 32 times in total. For the VE with either matched or mismatched scenario, we set the criteria as below:

- Matched sensory stimulus and description: All sensory components in the description rendered correctly in the virtual environment.

- Mismatched sensory stimulus and description: At least one component of the visual feedback with its related components (audio, tactile, smell) are missing in the virtual environment.

In Table 1, we provide the combination of the sensory component with the script in this study. For example, in the matched condition with scenario number 1 , the participant will see a helicopter with its engine sound in TVR, and the user will feel the engine via the floor vibrating with wind blowing and smelling a metallic (tar) scent in MVR. On the other hand, the helicopter and its related feedback (engine sound, metallic scent, the unique rhythmic vibration) will not be rendered in the unmatched condition in the same scenario.

\subsubsection{Hypotheses}

We expected that higher confidence levels and deeper immersion would occur in MVR since our system provides richer sensory feedback compared to TVR. Based on this expectation, we considered the following research hypotheses in this experiment:

$\mathrm{H}_{1}$ MVR will result in higher confidence levels and faster response times than a TVR.

$\mathrm{H}_{2}$ MVR and TVR will result in similar correct-answer ratios.

$\mathrm{H}_{3}$ MVR will result in more positive subjective feelings (higher presence, less cybersickness, higher preference) than TVR. 


$\rightarrow \begin{array}{ccc}\text { Informed Consent } \\ \text { and Demographic }\end{array} \rightarrow$ Instruction $\rightarrow$ Equip devices

Figure 5: Procedure flow for this experiment.

\subsection{Measurements}

\subsubsection{Confidence}

Since confidence is a subjective matter there is no perfect solution to measure it objectively. In our experiment, we directly asked the participant to report their confidence level with a 0 to 100 scale using a controller while they were immersed in VR. We assumed that strong confidence could spend less time for giving an answer. Based on the assumption, we recorded time lapse between the user entered to VE and completed reporting the confidence level. We also reported a correct answer ratio in this paper though we did not argue higher confidence will lead higher correct ratio in this experiment.

\subsubsection{Perceived Measures}

We measured two perceptual dependent variables, presence and cybersickness, using pre-validated questionnaires $[36,60]$, respectively. We conducted a brief post-exposure interview using a set of prompts ("Which VR experience did you prefer?", "Did you notice floor vibration?", "If so how was it?", "Did you notice any smells?", "If so, could you distinguish different smells?", "Did you notice any wind?", "If so, how was it?") for direct comparison purposes between the MVR and TVR after the participant completed all the experiment conditions. We first asked the subject for their preference, then continued the interview with rest of the questions.

\subsection{Procedures}

Before the experiment was conducted, each participant gave their informed consent and filled in demographic data. After completion of the demographics questionnaire, the participant was given instructions on how to use the controllers during the experiment as well as being given information on the structure of the experiment. They then entered the multi-sensory cage and sat on a chair before being given the HMD, headphones and a controller. Once the participant was ready the system was turned on and the participant was performed a test trial to ensure they understood the controls. Each trial consisted of two parts: Firstly, a darkened VR environment with a description of a scene and secondly a VR scene which may or may not correspond to the description given. Once the participant clearly understood the description they confirmed this by pulling the trigger on the controller. Upon pulling the trigger they were teleported into a VR location. The participant was then asked to determine whether the scene they were in matched the description they had been given. The participant provided their answer (yes or no) using the touchpad on the controller and a visualized choice interface (See Fig4 (a)), and confirmed their choice by pulling the trigger on the controller. The participant then reported the level of confidence in their choice using the touchpad on the controller and again confirmed their choice using the trigger. They were aided using a visualised vertical gauge bar in this step (See Fig4 (b)). Once the participant had confirmed their confidence level they were moved back into the darkened space with a description of the next scene. This was repeated sixteen times each condition for both multi-sensory and typical VR, excluding the test session. We provide a flow chart for this procerue in Figure 5. The participant was fixed in place in this study and was not able to
Table 3: Descriptive statistics.

\begin{tabular}{lllll} 
& Confidence & $\begin{array}{l}\text { Time duration } \\
\text { (second) }\end{array}$ & Presence & Preference \\
\hline \hline$p$-Value & $p<0.01$ & $p<0.06$ & $p<0.01$ & N/A \\
\hline Cohen's d & $d=-0.33$ & $d=0.17$ & $d=0.52$ & N/A \\
\hline MVR & $(0.83,0.18)$ & $(\mathbf{1 8 . 3 0}, 10.86)$ & $\mathbf{( 4 . 6 1 , 1 . 8 8 )}$ & $\mathbf{1 7 / 1 7}$ \\
$(M, S D)$ & & & & voted \\
\hline TVR & $(\mathbf{0 . 9 1}, 0.18)$ & $(16.60,9.09)$ & $(3.70,1.60)$ & $0 / 17$ voted \\
$(M, S D)$ & & & &
\end{tabular}

actively move around. Additionally, the participant was not provided with a virtual body.

As three different scents were used in this study, an odor neutralizer spray was used immediately after the scent was emitted to prevent it from lingering too long. After the participant completed the first session of trials, they were asked to fill out questionnaires. The study continued for a second set of trials after a 10 minute break for the participant. Upon completion of the second set of trials the participant was again asked to fill out questionnaires. Finally, the participant was subjected to an interview. In this interview the participants were asked about their system preference and any feedback on the multi-sensory stimuli.

\section{REsults}

In this section, we show results for the objective responses of the reported confidence level, time lapse, and correct answer ratio for answering in each trials, and subjective questionnaire responses for sense of presence, cybersickness, and user preference. For the analysis, we used a total of 17 participant data sets. We provide the interquartile range with outliers and median line in all box plots at the $95 \%$ level. We provide descriptive statistics in Table 3, and present the results for correct answer ratio and SSQ in Table 4 and 5 , respectively.

\subsection{Objective responses}

A Pearson product-moment correlation coefficient was computed to assess the relationship between the amount of time lapse that one consumed and rating of confidence level. There was a negative correlation between the two variables, $r=-0.28, n=544, p=0.01$. Overall, there was a weak, negative correlation between time lapse and confidence level. Increases in time lapse were correlated with decreased in rating of confidence level.

\subsubsection{Confidence Level}

We compared the two VR systems for self-reported confidence level using two-sample t-test with Welch's test because we dropped one participant's data partially. We confirmed the assumptions of the repeated measures with a Shapiro-Wilk test. Figure 6 shows the results in the form of box plot in which participants had to report the their confidential level on the answer which they made in VE with 0 to 100 scale. We found that the self-reported confidence level differed significantly between the two virtual reality system type ( $p=0.01$, Cohen's $d=-0.33$ ), and revealed a higher confidence for the TVR $(M=0.91, S D=0.18)$ than MVR $(M=0.83, S D=0.29)$.

\subsubsection{Elapsed Time for Answering}

We compared the two VR systems for time lapse for giving answering in each trial using repeated two sample t-test. Figure 7 shows the results in the form of box plot in which we recored the time duration between the participant entered to VE until they reported the confidence level. We found that the time consumption failed to reject the null hypothesis between the two virtual reality system type ( $p=0.06$, Cohen's $d=0.17$ ) but loosely support the difference still, 


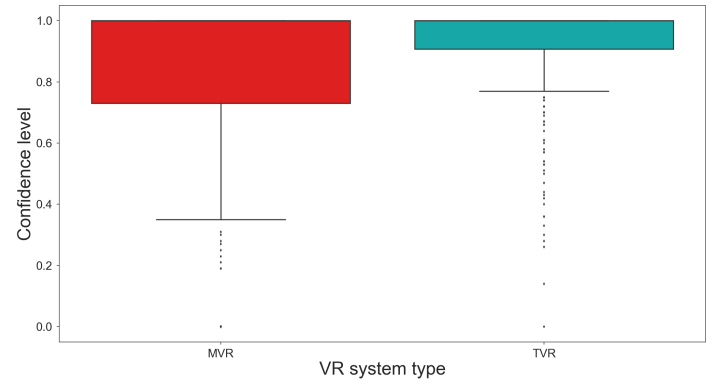

Figure 6: Results of the confidence level. TVR shows a higher confidence level than MVR. Due to the low complexity of the task, we found a ceiling effect from the result.

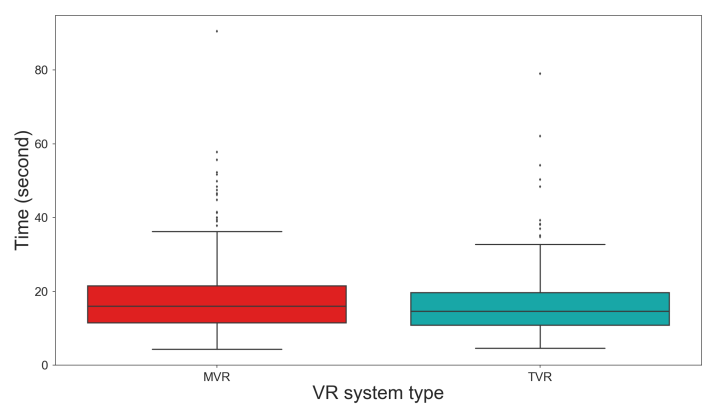

Figure 7: Results of the time consumption. The time lapse for the task completion in MVR was higher than TVR. Due to the more number of given sensory stimulus in MVR, the participant spent more process time.

and revealed a higher time spending for the MVR $(M=18.30 \mathrm{sec}$, $S D=10.86)$ than TVR $(M=16.60 \mathrm{sec}, S D=9.09)$.

\subsubsection{Correctness of the Answer}

Although TVR showed a slightly higher accuracy in terms of correct answer rate (we summed Hit and Correct rejection for the correctness and we had $80.47 \%$ in TVR and $79.30 \%$ in MVR), we did not find noticeable differences in forced-choice ratio from the both system (See Table 4).

\subsection{Subjective responses}

\subsubsection{Presence}

Since we adopted a set of pre-validated questions, we did not assess the construct validity again. To analyze the effects, we performed

Table 4: Correct answer ratio

\begin{tabular}{ll||ll}
\multicolumn{1}{c||}{} & \multicolumn{2}{c}{ Response } \\
\hline \hline \multirow{2}{*}{ MVR } & Matched & Yes & No \\
& Mismatched & FalseAlarm & Miss (1.56\%) \\
& & CorrectReject \\
& & $(19.14 \%)$ & $(31.25 \%)$ \\
\hline \hline TVR & Matched & Hit (47.66\%) & Miss (2.34\%) \\
& Mismatched & FalseAlarm & CorrectReject \\
& & $(17.19 \%)$ & $(32.81 \%)$
\end{tabular}

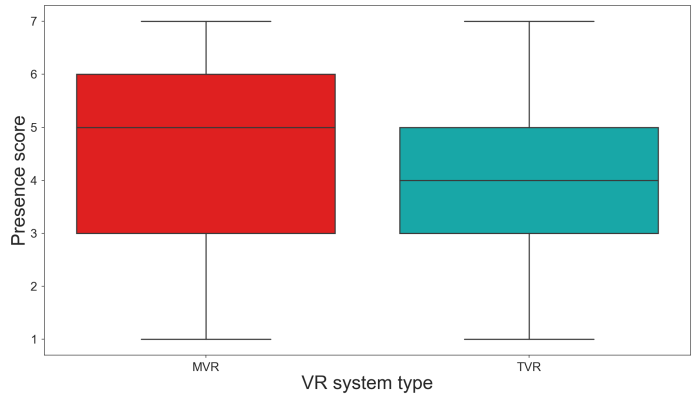

Figure 8: Results of the sense of presence. MVR shows a higher sense of presence than TVR.

Table 5: SSQ result. The maximum possible scores are 292 for disorientation, 200 nausea, 159 oculomotor, and 235 scaled total.

\begin{tabular}{lllll} 
& pre MVR & post MVR & pre TVR & post TVR \\
\hline \hline Disorientation & 109.72 & 120.64 & 105.17 & 122.19 \\
\hline Nausea & 72.95 & 81.62 & 73.14 & 84.27 \\
\hline Oculomotor & 60.19 & 68.64 & 59.80 & 66.96 \\
\hline Total & 87.78 & 98.28 & 86.44 & 98.90
\end{tabular}

Mann-Whitney U tests for all items at the 5\% significance level since the Anderson-Darling test rejected the hypothesis of normality at the $1 \%$ significance level in our data. Figure 8 shows the subjective questionnaire result that the sense of presence differed significantly between the two virtual reality system type ( $p=0.01$, Cohen's $d=0.52)$, and revealed a higher presence for the MVR $(M=4.61$, $S D=1.88)$ than TVR $(M=3.70 \mathrm{sec}, S D=1.60)$.

\subsubsection{Simulator Sickness Questionnaire}

We measured simulator sickness using a Kennedy's Simulator Sickness Questionnaire (SSQ), which asks the participant to score 16 symptoms with scores from 0 (none) to 3 (severe) [36]. These symptoms are categorized into three groups: Disorientation, Nausea, and Oculomotor. In this study, we did not find a noticeable difference between the VR systems, and both systems provoked a slight cybersickness. The Pre-SSQ score average over in MVR was 87.78 and the Post-SSQ score was 98.28, and the Pre-SSQ score average over in TVR was 86.44 and the Post-SSQ score was 98.90. Such SSQs for both increased indicated a range between slight-moderate [34]. We report the detailed SSQ scores in Table 5.

\subsubsection{Post-comparison Questionnaire}

At the end of the session, we asked each participant to choose which system was subjectively more immersive and preferred. All of the participants stated that the MVR made them feel as if they were in the virtual environment more immersively. Regarding system preferences, all participants preferred MVR over TVR as well. However, we have not yet measured this objectively.

\section{Discussion}

In this experiment, we found the benefits of the multi-sensory VR system over the typical-sensory VR system in terms of the sense of presence and user preference. The subjective questionnaire responses showed a significant effect that MVR elicited a higher sense of presence. Moreover, when asked for their preference, surprisingly, all participants indicated that they preferred MVR over TVR in the experiment. We expected MVR could reduce cybersickness 
since it could compensate for the sensory conflict [47], but we did not observe the difference in this study. Overall, we conclude that our finding partially supports hypothesis $\mathrm{H}_{3}$. However, participants showed higher confidence in their answer in the TVR system, although the presence and preference were superior for MVR. Also, we found the time duration was significantly higher in MVR. Thus, we cannot support our hypothesis $\mathrm{H}_{1}$.

We speculate that we observed multi-sensory feedback affected the participant's answers and on their confidence levels. Also, the participant incurred a higher cognitive processing load with the given sensory feedback to match compared to the prior information, scene description, in MVR, and it worked negatively in terms of confidence in our study. Another conjecture is that the complexity of the search and match task in the description was easy since we explicitly mentioned what the visual features should be found in the scene, so that the result of the correct answer ratio was both high and similar between the two conditions as we suggested in the hypothesis $\mathrm{H}_{2}$. Therefore, most participants could depend on the visual feedback primarily, and additional secondary feedback was not as important for this task.

From the results, we suspect that high-fidelity multi-sensory "rendering" of the virtual environment might not be appropriate for specific applications or tasks which require a low level of perception and cognition. In fact, less sensory feedback could be better for the tasks, since this avoids distracting the user. However, this does not mean TVR is the best solution for a decision-making scenario in complex environments. For this, we should conduct a study to explore this point of view in future work.

In addition, because of technical limitations (See Section 6), some participants were confused when they matched the secondary feedback with the primary feedback in MVR. Hence, we believe that if we deliver more sophisticated secondary feedback which matches better with the primary sensory feedback, then the user could have better confidence while they keep a superior sense of presence than the typical-sensory VR system. Based on the results from this experiment, overall, we would recommend that VR developers adopt the multi-sensory VR system in the area of training systems which require a high level of immersion, such as simulation of health-andsafety situations.

\section{Limitations}

Hardware We found some hardware-related technical limitations. First, the aroma dispensers used for the scent stimuli took over one second to heat up and deliver scent feedback resulting in a temporary mismatch between what the participant could smell and what they could perceive using their other senses. Also, some participants may have had difficulty identifying what smell was delivered, since smell is sometimes based on subjective experience. Second, some participant had difficulty identifying the vibration feedback for the helicopters and the waterfall although the wavelength of the sound source was completely different. Additionally, the controllers were unfamiliar to some of the participants and despite receiving some practice this unfamiliarity occasionally resulted in difficulties for the user when they attempted to give input.

Study In this study, we used a simple location-matching task with only a binary (Yes/No) answering system and the task environment might only induce low perceptual-cognitive load. We conjecture that our task environment could affect the user's responses, and that other environments may produce other results.

\section{CONCLUSION}

In this paper, we investigated the effects of a multi-sensory virtual reality system on confidence level, sense of presence and user preference in a virtual environment. We developed multiple sensory feedback enable experimental platform and provided vision, audio, floor vibration, wind blowing, and smell to the user in the platform, that is the state of the art technique in the world; no one developed such diverse level of sensory feedback and to our knowledge, this is the first approach to study the confidence level on the answering with multiple sensory feedback in virtual reality. We compared the multi-sensory VR condition with a typical-sensory VR, as a baseline condition. We found a significantly higher sense of presence and user preference with the multi-sensory VR system, but the confidence level was lower. We can think of two possible reasons for this. Either the task was too simplistic with insufficiently matched sensory feedback at a high level, or less sensory feedback was just better since it avoided sensory distractions while reporting the confidence level. To give a more clear answer, we would need to replicate the same experiment with only the visual feedback and compare it to a more sophisticated sensory feedback VR system to see if it improves, even more, the confidence levels.

\section{ACKNOWLEDGMENTS}

The material in this paper is based upon work supported in part by the HIT Lab NZ, University of Canterbury. Any opinions, findings, and conclusions or recommendations expressed in this material are those of the authors and do not necessarily reflect the views of the University of Canterbury.

\section{REFERENCES}

[1] P. Bach-Y-Rita, C. C. Collins, F. A. Saunders, B. White, and L. Scadden. Vision Substitution by Tactile Image Projection. Nature, 221(5184):963, Mar. 1969. doi: 10.1038/221963a0

[2] A. Bodnar, R. Corbett, and D. Nekrasovski. AROMA: ambient awareness through olfaction in a messaging application. pp. 183-190. ACM, 2004.

[3] M. Botvinick and J. Cohen. Rubber hands feel touch that eyes see. Nature, 391(6669):756-756, Feb. 1998. doi: 10.1038/35784

[4] D. A. Bowman and R. P. McMahan. Virtual reality: How much immersion is enough? Computer, 40(7):36-43, July 2007. doi: 10.1109/MC. 2007.257

[5] S. Brewster, D. McGookin, and C. Miller. Olfoto: designing a smellbased interaction. pp. 653-662. ACM, 2006.

[6] C. Burns. Razer Nari is a wireless headset with physical vibrations. SlashGear, Sept. 2018.

[7] R. M. Clifford, S. Jung, S. Hoermann, M. Billinghurst, and R. W. Lindeman. Creating a stressful decision making environment for aerial firefighter training in virtual reality. In Proceedings of the 26th IEEE Conference on Virtual Reality and 3D User Interfaces, IEEE VR 2019, 2019.

[8] H. Coelho, M. Melo, J. Martins, and M. Bessa. Collaborative immersive authoring tool for real-time creation of multisensory VR experiences. Multimedia Tools and Applications, Feb. 2019. doi: 10.1007/s11042 -019-7309-x

[9] S. B. Davis, G. Davies, R. Haddad, and M.-K. Lai. Smell me: Engaging with an interactive olfactory game. In People and Computers $X X$ Engage, pp. 25-40. Springer, 2007.

[10] C. Dietrich. Decision making: Factors that influence decision making, heuristics used, and decision outcomes. Student Pulse, 02, 2010.

[11] H. Q. Dinh, N. Walker, L. F. Hodges, C. Song, and A. Kobayashi. Evaluating the importance of multi-sensory input on memory and the sense of presence in virtual environments. pp. 222-228. IEEE, 1999.

[12] D. Dobbelstein, S. Herrdum, and E. Rukzio. inScent: a wearable olfactory display as an amplification for mobile notifications. pp. 130137. ACM, 2017.

[13] F. Drolet, M. Mokhtari, F. Bernier, and D. Laurendeau. A software architecture for sharing distributed virtual worlds. pp. 271-272. IEEE, 2009.

[14] M. Feng, A. Dey, and R. W. Lindeman. The effect of multi-sensory cues on performance and experience during walking in immersive virtual environments. In 2016 IEEE Virtual Reality (VR), pp. 173-174, Mar. 2016. doi: 10.1109/VR.2016.7504709

[15] M. Feng, A. Dey, and R. W. Lindeman. An initial exploration of a multi-sensory design space: Tactile support for walking in immersive 
virtual environments. In 2016 IEEE Symposium on 3D User Interfaces (3DUI), pp. 95-104, Mar. 2016. doi: 10.1109/3DUI.2016.7460037

[16] M. Feng, R. W. Lindeman, H. Abdel-Moati, and J. C. Lindeman. Haptic ChairIO: A system to study the effect of wind and floor vibration feedback on spatial orientation in VEs. In 2015 IEEE Symposium on $3 D$ User Interfaces (3DUI), pp. 149-150, Mar. 2015. doi: 10.1109/ 3DUI.2015.7131744

[17] S. Firestein. How the olfactory system makes sense of scents. Nature, 413(6852):211, 2001.

[18] M. Fontana, A. Dettori, F. Salsedo, and M. Bergamasco. Mechanical design of a novel Hand Exoskeleton for accurate force displaying. In 2009 IEEE International Conference on Robotics and Automation, pp. 1704-1709, May 2009. doi: 10.1109/ROBOT.2009.5152591

[19] F. Franz, E. Edgar, L. Albert-Georg, and B. Axel. G*power 3: A flexible statistical power analysis program for the social, behavioral, and biomedical sciences. Behavior Research Methods, 39(175-191), 2007

[20] A. Frisoli, F. Rocchi, S. Marcheschi, A. Dettori, F. Salsedo, and M. Bergamasco. A new force-feedback arm exoskeleton for haptic interaction in virtual environments. In First Joint Eurohaptics Conference and Symposium on Haptic Interfaces for Virtual Environment and Teleoperator Systems. World Haptics Conference, pp. 195-201, Mar. 2005. doi: 10.1109/WHC.2005.15

[21] G. Ghinea and O. Ademoye. The sweet smell of success: Enhancing multimedia applications with olfaction. ACM Transactions on Multime dia Computing, Communications, and Applications (TOMM), 8(1):2, 2012.

[22] D. Heeger and P. D. Heeger. Signal detection theory. In Univ., 2003 [Online]. Available: http://www.cns.nyu.edu/ david/sdt/sdt.html, 1997.

[23] Heilig, Morton L. Sensorama simulator, Jan. 1961.

[24] R. S. Herz. Aromatherapy facts and fictions: a scientific analysis of olfactory effects on mood, physiology and behavior. International Journal of Neuroscience, 119(2):263-290, 2009.

[25] S. Hoermann, E. A. Franz, and H. Regenbrecht. Referred Sensations Elicited by Video-Mediated Mirroring of Hands. PLoS ONE, 7(12):e50942, Dec. 2012. doi: 10.1371/journal.pone.0050942

[26] T. Huber, T. Wunderling, M. Paschold, H. Lang, W. Kneist, and C. Hansen. Highly immersive virtual reality laparoscopy simulation: development and future aspects. Int J CARS, 13(281-290), 2018.

[27] M. Ischer, N. Baron, C. Mermoud, I. Cayeux, C. Porcherot, D. Sander, and S. Delplanque. How incorporation of scents could enhance immersive virtual experiences. Frontiers in Psychology, 5, July 2014. doi: 10 3389/fpsyg. 2014.00736

[28] S. Jackson, Simon A.and Kleitman. Individual differences in decisionmaking and confidence: capturing decision tendencies in a fictitious medical test. Metacognition and Learning, 9(1):25-49, Apr 2014. doi: 10.1007/s11409-013-9110-y

[29] John P. Cater. Letters to the Editor: The Nose Have It! Presence: Teleoperators and Virtual Environments, 1(4):493-494, Jan. 1992. doi: 10.1162/pres. 1992.1.4.491

[30] L. Jones, C. A. Bowers, D. Washburn, A. Cortes, and R. V. Satya. The effect of olfaction on immersion into virtual environments. $\mathrm{Hu}$ man performance, situation awareness and automation: Issues and considerations for the 21st century, pp. 282-285, 2004.

[31] S. Jung and C. E. Hughes. The effects of indirect real body cues of irrelevant parts on virtual body ownership and presence. In ICAT-EGVE 2016 - International Conference on Artificial Reality and Telexistence and Eurographics Symposium on Virtual Environments, pp. 107-114, 2016

[32] S. Jung, C. Sandor, P. J. Wisniewski, and C. E. Hughes. Realme: The influence of body and hand representations on body ownership and presence. In Proceedings of the 5th Symposium on Spatial User Interaction, ACM SUI 2017, pp. 3-11, 2017.

[33] S. Jung, P. J. Wisniewski, and C. E. Hughes. In limbo: The effect of gradual visual transition between real and virtual on virtual body ownership illusion and presence. In Proceedings of the 25th IEEE Conference on Virtual Reality and 3D User Interfaces, IEEE VR 2018, 2018.

[34] B. Kaufmann, P. Kozeny, S. Schaller, J. N. A. Brown, and M. Hitz. May cause dizziness: Applying the simulator sickness questionnaire to handheld projector interaction. In Proceedings of the 26th Annual BCS Interaction Specialist Group Conference on People and Computers, BCS-HCI '12, pp. 257-261. British Computer Society, Swinton, UK, UK, 2012.

[35] J. N. Kaye. Symbolic olfactory display. PhD thesis, Massachusetts Institute of Technology, 2001.

[36] R. S. Kennedy, N. E. Lane, K. S. Berbaum, and M. G. Lilienthal. Simulator sickness questionnaire: An enhanced method for quantifying simulator sickness. The International Journal of Aviation Psychology, 3(3):203-220, 1993. doi: 10.1207/s15327108ijap0303

[37] K. Kilteni, A. Maselli, K. P. Kording, and M. Slater. Over my fake body: Body ownership illusions for studying the multisensory basis of ownbody perception. Frontiers in Human Neuroscience, 9(4), MARCH 2015.

[38] E. Kruijff, A. Marquardt, C. Trepkowski, J. Schild, and A. Hinkenjann. Designed emotions: challenges and potential methodologies for improving multisensory cues to enhance user engagement in immersive systems. The Visual Computer, 33(4):471-488, Apr. 2017. doi: 10. 1007/s00371-016-1294-0

[39] E. Kruijff, C. Trepkowski, and R. W. Lindeman. The Effect of Vibration and Low-frequency Audio on Full-body Haptic Sensations. In Proceedings of the 21st ACM Symposium on Virtual Reality Software and Technology, VRST '15, pp. 194-194. ACM, New York, NY, USA, 2015. event-place: Beijing, China. doi: 10.1145/2821592.2821626

[40] R. W. Lindeman, R. Page, Y. Yanagida, and J. L. Sibert. Towards Fullbody Haptic Feedback: The Design and Deployment of a Spatialized Vibrotactile Feedback System. In Proceedings of the ACM Symposium on Virtual Reality Software and Technology, VRST '04, pp. 146-149. ACM, New York, NY, USA, 2004. event-place: Hong Kong. doi: 10. 1145/1077534.1077562

[41] R. W. Lindeman, Y. Yanagida, H. Noma, and K. Hosaka. Wearable vibrotactile systems for virtual contact and information display. Virtual Reality, 9(2):203-213, Mar. 2006. doi: 10.1007/s10055-005-0010-6

[42] V. M. Manghisi, M. Fiorentino, M. Gattullo, A. Boccaccio, V. Bevilacqua, G. L. Cascella, M. Dassisti, and A. E. Uva. Experiencing the Sights, Smells, Sounds, and Climate of Southern Italy in VR. IEEE Computer Graphics and Applications, 37(6):19-25, Nov. 2017. doi: 10 .1109/MCG.2017.4031064

[43] J. Martins, R. Gonalves, F. Branco, L. Barbosa, M. Melo, and M. Bessa. A multisensory virtual experience model for thematic tourism: A Port wine tourism application proposal. Journal of Destination Marketing \& Management, 6(2):103-109, June 2017. doi: 10.1016/j.jdmm.2017. 02.002

[44] T. Nakamoto and H. P. D. Minh. Improvement of olfactory display using solenoid valves. pp. 179-186. IEEE, 2007.

[45] T. Nakamoto and K. Yoshikawa. Movie with scents generated by olfactory display using solenoid valves. IEICE transactions on fundamentals of electronics, communications and computer sciences, 89(11):33273332, 2006.

[46] M. Ponder, B. Herbelin, T. Molet, S. Schertenlieb, B. Ulicny, G. Papagiannakis, N. Magnenat-Thalmann, and D. Thalmann. Immersive vr decision training: Telling interactive stories featuring advanced virtual human simulation technologies. In Proceedings of the Workshop on Virtual Environments 2003, EGVE '03, pp. 97-106. ACM, New York, NY, USA, 2003. doi: 10.1145/769953.769965

[47] J. T. Reason and J. J. Brand. Motion sickness. Academic press, 1975.

[48] H. Regenbrecht, S. Hoermann, C. Ott, L. Mller, and E. Franz. Manipulating the Experience of Reality for Rehabilitation Applications. Proceedings of the IEEE, 102(2):170-184, Feb. 2014. doi: 10.1109/ JPROC.2013.2294178

[49] E. Richard, A. Tijou, P. Richard, and J.-L. Ferrier. Multi-modal virtual environments for education with haptic and olfactory feedback. Virtual Reality, 10(3-4):207-225, 2006.

[50] J. Sato, K. Ohtsu, Y. Bannai, and K.-i. Okada. Effective presentation technique of scent using small ejection quantities of odor. pp. 151-158. IEEE, 2009.

[51] V. Schwind, P. Knierim, N. Haas, and N. Henze. Using presence questionnaires in virtual reality. In Proceedings of the 2019 CHI Conference on Human Factors in Computing Systems, CHI '19, pp. 360:1-360:12. 
ACM, New York, NY, USA, 2019. doi: 10.1145/3290605.3300590

[52] R. K. Selander, C. D. W. Brooks, and L. M. Popplewell. Fragrance delivery for multimedia systems, 2006.

[53] A. Skulmowski, A. Bunge, K. Kaspar, and G. Pipa. Forced-choice decision-making in modified trolley dilemma situations: a virtual reality and eye tracking study. Frontiers in behavioral neuroscience, 8 , 2014.

[54] M. Slater, J. McCarthy, and F. Maringelli. The influence of body movement on subjective presence in virtual environments. Human Factors, 40(3):469-477, 1998. PMID: 9849105. doi: 10.1518/ 001872098779591368

[55] M. Slater and M. V. Sanchez-Vives. Enhancing our lives with immersive virtual reality. Frontiers in Robotics and AI, 3:74, 2016. doi: 10. 3389/frobt.2016.00074

[56] C. Summerfield 1 and F. P. de Lange. Expectation in perceptual decision making: neural and computational mechanisms. Nature Reviews Neuroscience, 15:745-756, 2010.

[57] J. Thar, S. Stoenner, and J. Borchers. HapticPong: Low Resolution Games for Visual Impaired. In Proceedings of the 2018 Annual Symposium on Computer-Human Interaction in Play Companion Extended Abstracts - CHI PLAY '18 Extended Abstracts, pp. 133-136. ACM Press, Melbourne, VIC, Australia, 2018. doi: 10.1145/3270316. 3270593

[58] J. Thar, S. Stoenner, and J. Borchers. HaptiGames - Personally Fabricated for Visual Impaired. In Proceedings of the 2018 Annual Symposium on Computer-Human Interaction in Play Companion Extended Abstracts - CHI PLAY '18 Extended Abstracts, pp. 137-141. ACM Press, Melbourne, VIC, Australia, 2018. doi: 10.1145/3270316. 3270592

[59] R. Tortell, D. P. Luigi, A. Dozois, S. Bouchard, J. F. Morie, and D. Ilan. The effects of scent and game play experience on memory of a virtual environment. Virtual Reality, 11(1):61-68, 2007.

[60] M. Usoh, E. Catena, S. Arman, and M. Slater. Using presence questionnaires in reality. Presence: Teleoper. Virtual Environ., 9(5):497-503, Oct. 2000. doi: 10.1162/105474600566989

[61] D. A. Washburn and L. M. Jones. Could olfactory displays improve data visualization? Computing in Science \& Engineering, 6(6):80, 2004.

[62] Y. Yanagida, S. Kawato, H. Noma, A. Tomono, and N. Tesutani. Projection based olfactory display with nose tracking. In IEEE Virtual Reality 2004, pp. 43-50, Mar. 2004. doi: 10.1109/VR.2004.1310054

[63] Y. Ye and A. Steed. Is the rubber hand illusion induced by immersive virtual reality? In In IEEE Conference on Virtual Reality, pp. 95-102, 2010. 